\title{
Genetic Algorithm Based Modeling of Doubly-Fed Wind Farms
}

\author{
Yujia $\mathrm{Gu}^{1}$, Yinfeng Wang ${ }^{2}$, Xutao $\mathrm{Li}^{1}$, Bei $\operatorname{Tian}^{1}$, Feng Gao and Chao $\mathrm{Lu}^{2, *}$ \\ ${ }^{1}$ Power Science Research Institute of State Grid Ningxia Power Co., Yinchuan, China 750011 \\ ${ }^{2}$ Tsinghua University, Beijing, China 100084 \\ ${ }^{*}$ Corresponding author
}

\begin{abstract}
Faced with the drawback of conventional doubly-fed wind farm models, which cannot reliably reflect the actual control law and dynamic characteristics, a method of complex characteristics modeling based on simulation is developed in this paper. The software MATLAB/Simulink platform is utilized to create a universal equivalent model, including structure characters and control modes. In order to make the parameters identification process of higher-order wind farm models simplifier, trajectory sensitivity analysis method is used to select dominant dynamic parameters. Besides, a method melting the bright side of model simulation and the genetic algorithm is proposed to search the optimal parameter combination. The test results on the IEEE 9-bus system demonstrate the effectiveness of the proposed comprehensive method.
\end{abstract}

Keywords-doubly-fed wind farm; matlab/simulink; trajectory sensitivity analysis; model simulation; genetic algorithm

\section{INTRODUCTION}

In recent years, wind energy resources get the favor of people because of its advantages such as rich reserves, environmental protection and flexible application. However, the fluctuation and randomness features of wind power are especially evident, as increasement of wind power penetration in power system. Thus, establishing the model that can effectively reflect the actual characteristics of wind farms and reliably setting the model parameters are both important to analyze the impact of large scale wind power integration into power systems. Previously, in order to study the faults in a large-scale wind farm affected by complex relationship among those doubly-fed wind generators, the wind generators are classified by dynamic consistency and the aggregated to get the output features. Actually, the wind farms internal structure is complex and changeable. The overall output features have more influence on the system safe and stable operation in the engineering practice. Therefore, the research work of reliability modeling of large-scale wind farms carried out recently are more focused on the external response properties of overall wind farms.

In allusion to the difficulty that the high-order models are too complex to perform efficient parameter identification. The majority of modeling methods already proposed [1,2] would simplify the order of equivalent wind farm models according to different research problems. Although the model complexity is greatly decreased by this mean, some control laws and response characteristics of the wind farms may be lost. So the scope of those methods is restricted. In the process of wind farm modeling, there also has been a special outpouring of concern for the model parameters selection methods that are commonly used in the daily analysis and calculation of dispatch and operation departments. Setting unified and typical parameters for all the wind farms in power system is unreasonable. The above conditions show that modeling and parameter identification based on measured data is imperative. To overcome such limitation, a modeling and optimized parameter identification method of doubly-fed wind farms based on MATLAB/Simulink is proposed in this paper. on the basis of G.E.'s 1.5/3.6MW doubly fed induction generator (DFIG) models [3], an equivalent model of wind farms is established in Simulink platform, which includes fundamental control links and response characteristics. In addition, the parameters with high trajectory sensitivities related to output active and reactive powers are treated as the dominant parameters. Genetic algorithm is embedded in the process of model simulation to obtain the optimized parameter combination, which avoids the cumbersome process of model simplification and offers an effective solution to the parameters optimization of complex models. A comparison between the simulation results with optimal and actual parameters in WECC 9-bus system is made to validate the effectiveness and reliability of the modeling methodology.

\section{MODEL ESTABLISHMENT OF WIND FARMS}

The model presented in this article describes the relevant dynamics of doubly-fed wind farms in the $s$ domain, which consists of four parts: the equivalent shaft model, active power control model, reactive control model and grid interface model. The overall connectivity of this model can be observed in Figure I.

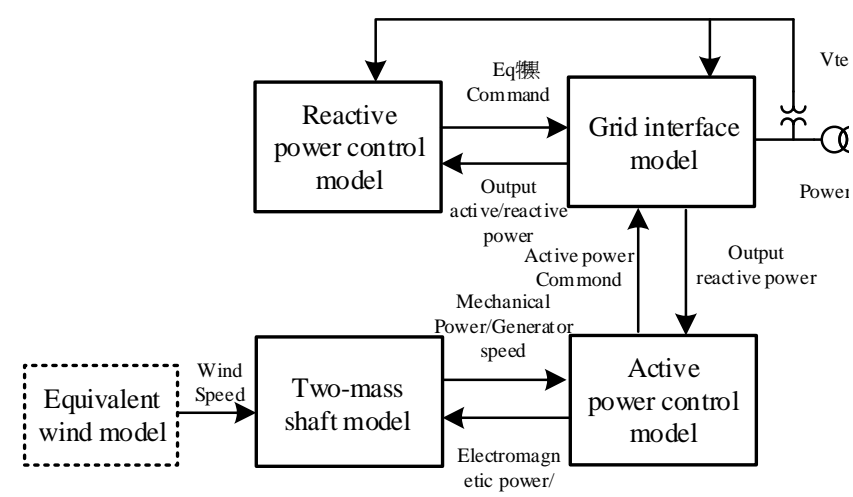

FIGURE I. DYNAMIC MODEL CONNECTIVITY OF DOUBLY-FED WIND FARMS. 


\section{A. Equivalent Shaft Model}

Because there is a considerable difference between the moments of inertia of the wind turbine's drive shaft and the generator rotor, two masses are separately equivalent to the two connected parts, as detailed in Figure II. The wind turbine, low speed shaft and gear box are converted into the equivalent mass model at low speed side, with their details ignored. Rigidity coefficient and damping coefficient are included in the model to give a better view of the characteristics of the wind turbine and generator mechanical structure.

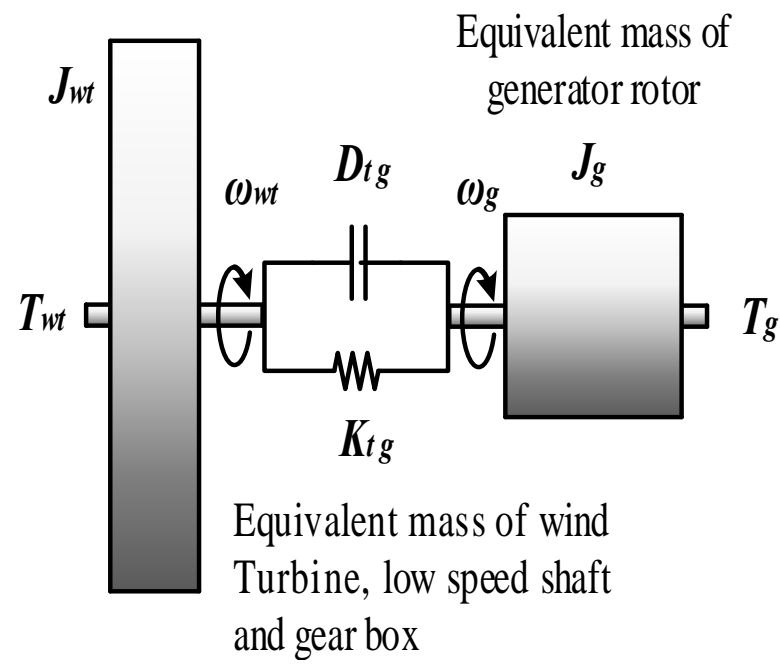

FIGURE II. TWO-MASS SHAFT MODEL OF WIND FARMS.

\section{B. Active Power Control Model}

Now most of the main wind farms adopt variable-speed constant-frequency (VSCF) technology. The pitch angle of wind turbine is adjusted to achieve the VSCF control strategy under various wind speeds. Besides, the equivalent power absorbed by the wind turbine can be calculated by equation, as in (1).

$$
P_{\text {wind }}=\frac{1}{2} \rho S_{r} C_{p}(\theta, \lambda) V_{\text {wind }}^{3}
$$

Where $P_{\text {wind }}$ is the equivalent active power of the overall wind farm. $\rho$ is the average density of air. $S_{r}$ is the equivalent windward area. $V_{\text {wind }}$ is the instantaneous average wind speed. $C_{p}$ is called wind power utilization factor, which is the function of pitch angle $\theta$ and tip speed ratio $\lambda$.

Since tip speed ratio is the ratio of wind speed and linear velocity of blade tip. It is apparent that pitch angle control plays an important role in active power control. The control system block diagram is displayed in Figure III.

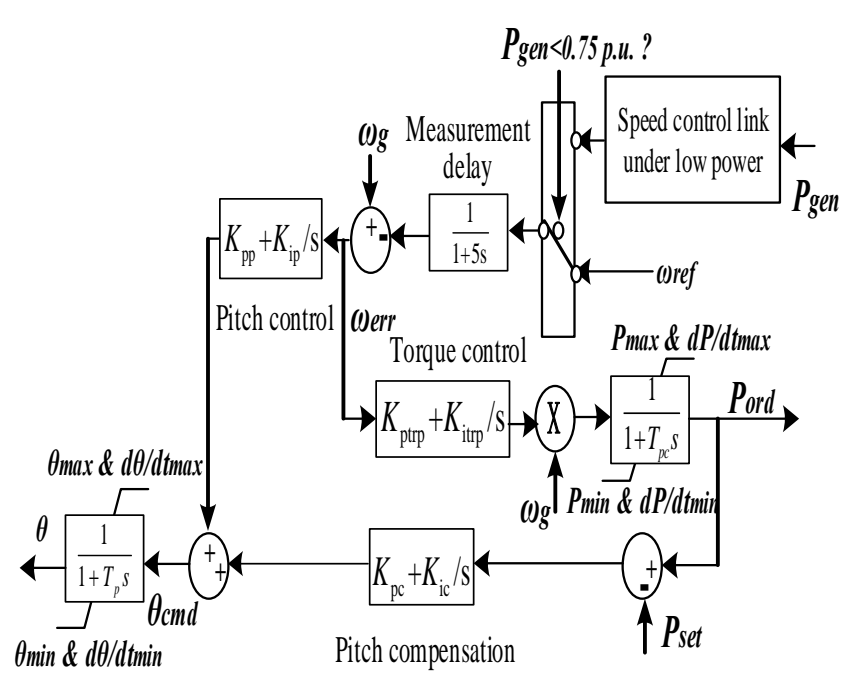

FIGURE III. PICTH ANGLE CONTROL DIAGRAM.

Generally, the reference speed of generator rotor is 1.2p.u. However, it is recalculated by the link of speed control under low power, when the output active power is lower than 0.75 p.u. Besides, the saturation modules and the rate limiters are set to simulate the limitation of dynamic changing ability of the pitch angle and electromagnetic power.

\section{Reactive Power Control Model}

The reactive power is controlled with closed-loop control strategy in this model. By monitoring magnitude of the terminal voltage and the reactive power, the controller compares them against their references. PI regulators and time delayers are given to improve the performance of this controller. The control mode is alternative as required, choosing between constant voltage control and constant power factor control. The most difference between them is the control objective. So different calculation processes of reactive reference are used, as in (2) and (3).

$$
Q_{o r d 1}(s)=P_{g e n}(s) \cdot e^{-s \cdot T_{p m}} \cdot \tan (\arccos (P F))
$$

$$
\begin{aligned}
& V_{c}(s)=\left|V_{\text {term }}(s)-Z_{c} \cdot I_{g e n}(s)\right| \cdot e^{-s \cdot T r} \\
& Q_{\text {ord } 2}(s)=\left[V_{\text {ref }}-V_{c}(s)\right] \cdot\left(K_{p v}+K_{i v} / s\right) \cdot e^{-s \cdot T_{V}}
\end{aligned}
$$

Where $P F$ is the power factor. $P_{\text {gen }}$ is the measure value of the wind farm active power. Time constant $T_{p m}$ and $T_{r}$ represent the measurement lag. $T_{v}$ is the communication delay. $V_{\text {term }}$ is the terminal voltage of the wind farm. $Z_{c}$ is the equivalent impedance of the collector system in wind farm. Igen is the output current. $V_{c}$ is the calculated value of generator terminal voltage. While $V_{r e f}$ is the reference value. $K_{p v}$ and $K_{i v}$ are Proportional and integral coefficients of the PI controller. Qord 1 is the reactive power command value of 
constant power factor control. Similarity, Qord2 represents the command value of constant reactive power.

\section{Grid Interface Model}

As it is indicated in the Figure IV, the whole wind farm is integrated into the power grid as a controlled current source. $X_{e q}$ is the equivalent reactance of the whole wind farm . Active and reactive current are respectively controlled by active command and quadratyre axis transient electromotive force. This model simulates the connection among the converter, generator stator and the power grid.

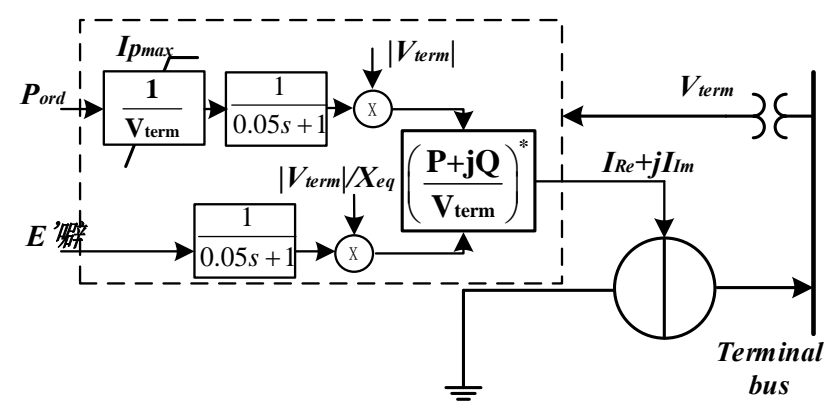

FIGURE IV. GRID INTERFACE MODEL DIAGRAM.

\section{MODEL PARAMETERS IDENTIFICATION BASED ON SIMULATION}

Few researchers have addressed the problem of parameters identification of the complex wind farm models. It's difficult to make a balance between the model's accuracy and the parameter identifiability. The purpose of this study is to optimize the parameter combination, which the simulation results based on correspond with the actual dynamic response results as possible.

\section{A. Selection of the Domination Parameters}

As mentioned previously, the main difficulty of parameter identification is too many parameters. The parameters of PI controller, saturation and rater limiter can be off-line set, without frequent exchange. The other parameters with clear physical meanings need to be identified, as listed in Table I.

TABLE I. PARAMETERS AND THEIR TYPICAL VALUES OF WIND FARM.

\begin{tabular}{|c|c|c|}
\hline Parameter name & Symbol & $\begin{array}{l}\text { Typical } \\
\text { (p.u.) }\end{array}$ \\
\hline $\begin{array}{c}\text { Equivalent reactance of the wind } \\
\text { farm }\end{array}$ & Xeq & 0.55 \\
\hline Innertial time constant of generator & $T g$ & 1.14 \\
\hline $\begin{array}{c}\text { Innertial time constant of wind } \\
\text { turbine }\end{array}$ & $T w t$ & 7.64 \\
\hline Rigidity coefficient of the shaft & Kgt & 1.25 \\
\hline Damping coefficient of the shaft & $\mathrm{Dgt}$ & 1.50 \\
\hline
\end{tabular}

As we know, those parameters that are most sensitive to the output are more easily identified [4], [5]. So the trajectory sensitivity defined in (4), is used to analyze the identifiability of those parameter.

$$
S_{\theta_{i}}=\lim _{\Delta \theta_{i} \rightarrow 0} \frac{\frac{y\left(t, \theta, \theta_{i 0}+\Delta \theta_{i}\right)-y_{0}\left(t, \theta, \theta_{i 0}\right)}{y_{0}\left(t, \theta, \theta_{i 0}\right)}}{\Delta \theta_{i} / \theta_{i 0}}
$$

Where $S_{\theta_{i}}$ is the trajectory sensitivity of the ith parameter $\theta_{i}$ related to the output variable $y . y_{0}$ is the initial value of $y$, as an observational variable. when the $\theta_{i}$ is given as $\theta_{i 0}$. $\Delta \theta_{i}$ is the variable quantity of the parameter $\theta_{i} . \theta$ includes all the parameters except for $\theta_{i}$.

The trajectory sensitivity is change over time in the dynamic process. For ease of comparison among those parameters, the average values can be calculated in the response time periods, as in (5).

$$
\overline{S_{\theta i}}=\frac{1}{t_{1}-t_{0}} \int_{t_{0}}^{t_{1}} S_{\theta i}(t) d t
$$

Where the $t_{0}, t_{1}$ are separately the start and end times of the dynamic process. $S_{\theta i}(t)$ is the trajectory sensitivity of parameter $\theta_{i}$ at time $t . \overline{S_{\theta i}}$ is the average value. This equation can be also expressed as a discrete form.

Above all, the output active and reactive power of the wind farm model are selected as observational variables. The average trajectory sensitivities of those parameters related to the two observational variables are used to determine the dominant parameters.

\section{B. The Optimization Algorithm Based on Simulation}

The essence of the parameter identification is optimization under the limited conditions. For the wind model with complex structure and numerous control units, model simplification is meaningless to replicate the work of previous authors. So a method is timely developed in this section, which is not dependent on the model structure information, but the results of model simulation.

Firstly, an optimal model with constraint is built in (6). The optimization object is to minimize the error between the simulation value and the measured value of the model output. In addition, the parameters and the intermediate variables must vary in their limited ranges.

$$
\begin{array}{ll}
\min & \sum_{n=0}^{N}\left|\hat{Y}_{n}(u, \hat{\theta})-Y_{n}(u, \theta)\right|^{2} \\
\text { s.t. } & \theta_{\min } \leq \hat{\theta} \leq \theta_{\max } \\
& x_{\min } \leq \hat{x}(\hat{\theta}) \leq x_{\max }
\end{array}
$$


Where $Y_{n}(u, \theta)$ is the $n$th measured value of the observational variable with input variable $u$ and parameter combination $\theta$. While the $\hat{Y}_{n}(u, \hat{\theta})$ is the $n$th simulated value with the estimated value $\hat{\theta} . \hat{x}(\hat{\theta})$ is the estimates of the intermediate variable. $\mathrm{N}$ is the number of samples.

In order to make full use of the output information of the wind farm model, and as far as possible to reduce dependence on the model, the genetic algorithm is applied in the model parameter identification process. On the one hand, model simulation provides data for optimization algorithm. On the other hand, optimization algorithm is employed to search for the optimal parameters in the feasible region of the parameters. And then the parameters passed to the simulation model. The flow chart is as follows, in Figure V.

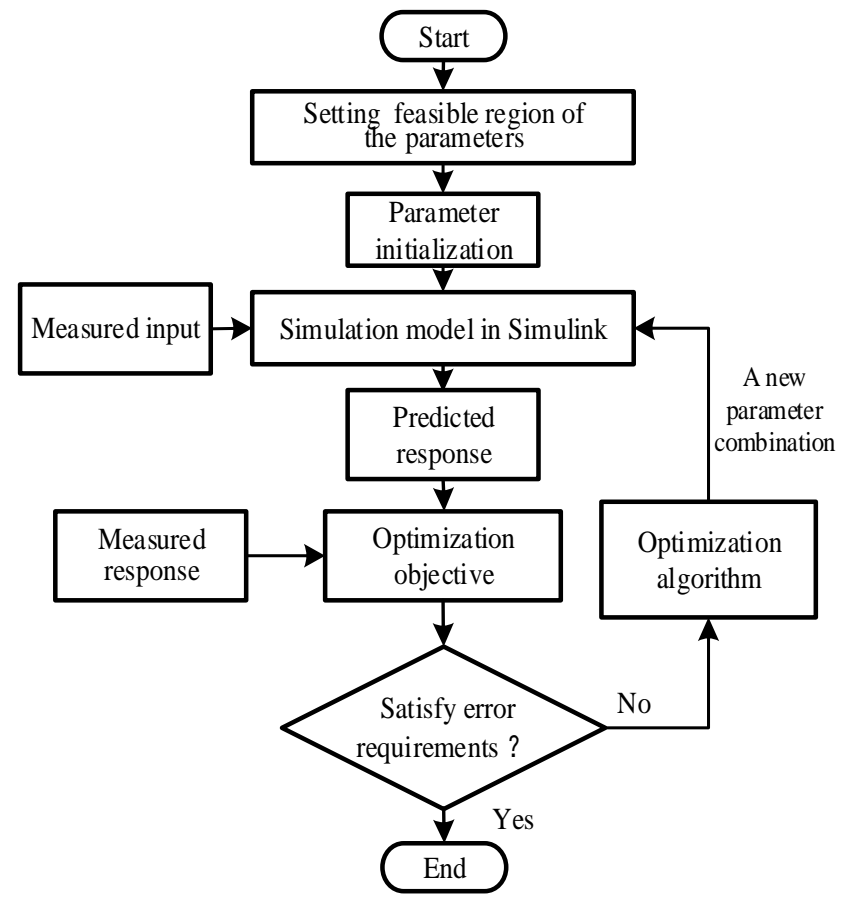

FIGURE V. THE FLOW CHART OF PARAMETER OPTIMIZATION.

The response data from the model simulation based on the current parameters is used to calculated the current value of objective function. The reciprocal of the objective function is set as double fitness, considering non-negative and minimization of the objective function. Furth more, the ratios between the single fitness and the total fitness act as the selection probability of each combination. It is apparent that the combination with high fitness would have a high probability of heredity to the next generation. Genetic crossover operation and mutation operation are applied to produce new parameter combinations with higher fitness. Then the parameter combination with highest fitness is delivered back to the simulation model. Model simulation and parameter optimization algorithm are alternately conducted till the response results satisfy the error requirements.

\section{ALGORITHM VERIFICATION FOR THE WIND FARM MODEL}

A test is carried out in the IEEE 9-bus system to demonstrate the effectiveness of the model and the algorithm, as in Figure VI. The generator GEN-1 is a slack generator, and the GEN-2 is a PV generator. While the GEN-3 is the doublyfed wind farms, which includes 67 wind generators. The system standard capacity is 100MVA.

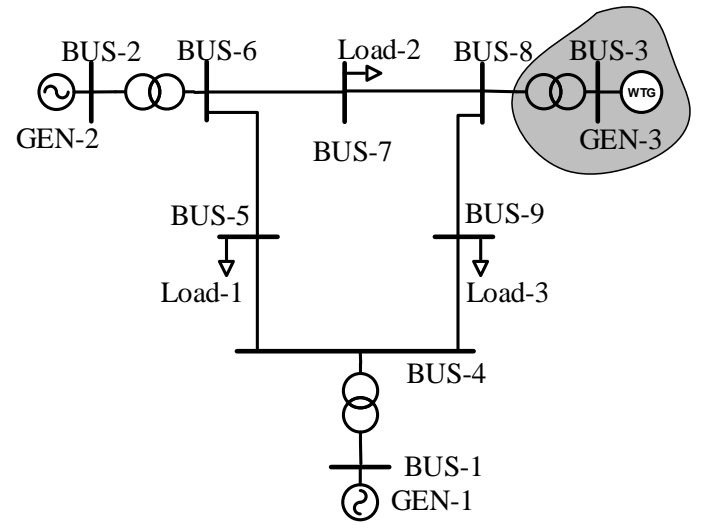

FIGURE VI. IEEE 9-BUS TEST SYSTEM.

The voltage of BUS-3 and the wind speed are selected as the input variables. The active power and the reactive power of the wind farm act as the observational variable. Two cases are studied in this test. The first one is that a three-phase short circuit fault is set at BUS-6 and continues 0.2 seconds. An $\mathrm{d}$ the other is the gust disturbance from 2.0s to 12.0 s. Simulation data is used to imitate the PMU data, restricted to the current conditions. The averages of trajectory sensitivity are summarized in Table II.

TABLE II. AVERAGES OF TRAJECTORY SENSITIVITY.

\begin{tabular}{|c|c|c|c|c|c|c|}
\hline Disturbance & $\begin{array}{c}\text { Observatio } \\
\text { n variables }\end{array}$ & $\boldsymbol{X e q}$ & $\boldsymbol{T g}$ & $\boldsymbol{T w t}$ & $\boldsymbol{K} \boldsymbol{t g}$ & $\boldsymbol{D t g}$ \\
\hline \multirow{2}{*}{$\begin{array}{c}\text { Short-circuit } \\
\text { fault }\end{array}$} & $P g$ & 0.187 & 0.020 & 0.006 & 0.012 & 0.001 \\
\cline { 2 - 7 } & $Q g$ & 0.558 & 0.104 & 0.056 & 0.030 & 0.003 \\
\hline $\begin{array}{c}\text { Gust } \\
\text { disturbance }\end{array}$ & $P g$ & 0.002 & 0.050 & 0.041 & 0.039 & 0.001 \\
\cline { 2 - 7 } & $Q g$ & 0.159 & 0.049 & 0.043 & 0.240 & 0.001 \\
\hline
\end{tabular}

The results in Table II show that the parameters (such as Xeq) with close links to the electrical parts have higher trajectory sensitivity under the fault disturbance. But on the contrary, the parameters (such as $\mathrm{Tg}, \mathrm{Kwt}, \mathrm{Ktg}$ ) related to the mechanical parts are more sensitive under the gust disturbance. Certainly, there are some parameter that is difficult to identify, Dtg is the one in this two cases.

Based on the analysis above, those parameters are divided into two groups to identify. The $X e q$ is identified in the fault disturbance case. The others including $\mathrm{Tg}, \mathrm{Kwt}, \mathrm{Ktg}$, are in the gust disturbance case. The response results of the measurement and simulation are compared in the Figure VII. There is only the comparison of active power owing to space reasons. 

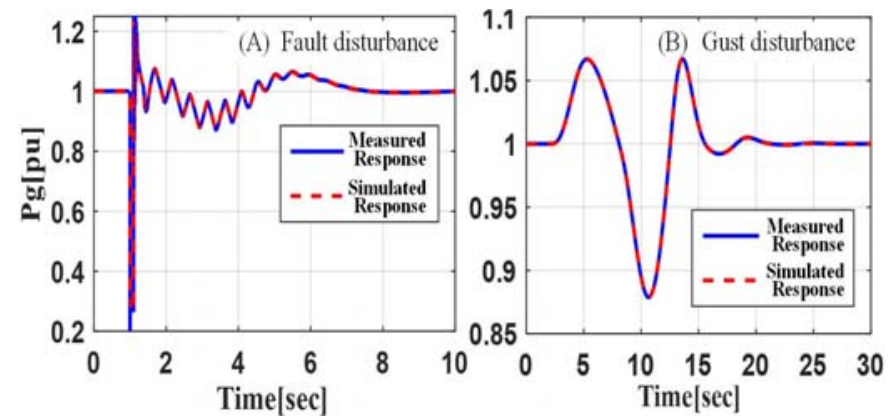

FIGURE VII. COMPARISION OF THE MEASURED RESPONSE AND MEASURED RESPONSE.

The parameter values of identification based on simulation almost corresponds to the actual values, which proves that the validity and practicability of the parameter identification method based on simulation, as detailed in the Table III.

TABLE III. COMPARISON OF PARAMETER ACTUAL VALUES AND INDENTIFIED VALUES.

\begin{tabular}{|c|c|c|c|c|}
\hline Parameter & $\boldsymbol{X e q}$ & $\boldsymbol{T g}$ & $\boldsymbol{T w t}$ & $\boldsymbol{K t g}$ \\
\hline Actual value & 0.520 & 1.200 & 7.600 & 1.250 \\
\hline Identified value & 0.518 & 1.183 & 7.511 & 1.267 \\
\hline Relative error & $0.38 \%$ & $1.42 \%$ & $1.17 \%$ & $1.36 \%$ \\
\hline
\end{tabular}

\section{CONCLUSIONS}

In summary, a new method based on simulation is presented to solve the modeling problem of doubly-fed wind farms in this paper. Dominant parameters and identifiability conditions are obtained by trajectory sensitivity analysis. Additionally, optimization algorithm is employed during the model simulation to search for the optimal parameter combination that can accurately reflect the dynamic characteristics of wind farms. Verification experiment in the IEEE 9-bus system provides compelling evidence of important application value of the method. Further work would focus on the improvement of computation efficiency of the method.

\section{ACKNOWLEDGMENT}

This work was supported in part by Major State Basic Research Development Program of China (2012CB215206) and National Natural Science Foundation of China (51037002 and 51107061).

\section{REFERENCES}

[1] L. Cheng, J. Lin and Y. Sun, "A Model for Assessing the Power Variation of a Wind Farm Considering the Outages of Wind Turbines," Sustainable Energy, IEEE Transactions on,vol. 3, pp. 432-444, 2012.

[2] R. Ye, H. Lin, Z. Chen, and Q. Gao, “Comparison of transient behaviors of wind turbines with DFIG considering the shaft flexible models,"in Electrical Machines and Systems, Wuhan, 2008, pp. 2585-2590.

[3] N. W. Miller, W. W. Price and J. J. Sanchez-Gasca, "Modeling of GE wind turbine-generators for grid studies', New York: General Electric International, Inc. 2004, pp.6-24.

[4] P. Ju, The theory and method of electrical power system modeling. Beijing: Science Press, 2010.
[5] H. Xie, P. Ju, and J. Luo, "Identifiability analysis of load parameter based on sensitivity calculation,"Automation of Electric Power Systems, vol. 33, pp. 17-21, 2009. 